\title{
Changements de rythme chez les Gnawa du Maroc
}

Le rituel et la scène

Between Ritual and Stage: The Changing Rhythm of Moroccan Gnawa Music

\section{Zineb Majdouli}

\section{(2) OpenEdition}

\section{Journals}

Édition électronique

URL : https://journals.openedition.org/clo/2031

DOI : $10.4000 /$ clo.2031

ISSN : 2266-1816

Éditeur

INALCO

Édition imprimée

Date de publication : 1 janvier 2013

ISBN : 978-2-85831-217-7

ISSN : 0396-891X

Référence électronique

Zineb Majdouli, «Changements de rythme chez les Gnawa du Maroc », Cahiers de littérature orale [En ligne], 73-74 | 2013, mis en ligne le 11 mai 2015, consulté le 01 juillet 2021. URL : http:// journals.openedition.org/clo/2031 ; DOI : https://doi.org/10.4000/clo.2031

Ce document a été généré automatiquement le 1 juillet 2021

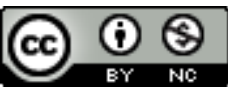

Cahiers de littérature orale est mis à disposition selon les termes de la Licence Creative Commons Attribution - Pas d'Utilisation Commerciale 4.0 International. 


\section{Changements de rythme chez les Gnawa du Maroc}

Le rituel et la scène

Between Ritual and Stage: The Changing Rhythm of Moroccan Gnawa Music

\section{Zineb Majdouli}

1 La cérémonie rituelle des Gnawa du Maroc se nomme lila ou littéralement «nuit ». Réservée a priori aux initiés, elle se caractérise, comme de nombreux rites à but thérapeutique, par des phases musicales, sensorielles et sociales inscrites dans une temporalité spécifique. Les participants à la lila remplissent leurs obligations rituelles, ravivent et réaffirment les alliances ${ }^{1}$ avec les saints musulmans et les entités invisibles (invoqués en vue des transes de possession) tout en célébrant leurs valeurs communes. La communauté confrérique des Gnawa, issue de la traite négrière en terre musulmane, pratique donc cette religion syncrétique mêlant à l'islam des références religieuses subsahariennes. Si les données sociohistoriques sur les origines de cette communauté restent imprécises ${ }^{2}$, l'usage courant du mot Gnawa fournit des informations précieuses sur le statut symbolique de ses membres. Ce terme est en effet largement péjoratif et désigne, dans l'imaginaire populaire, un individu noir, pauvre, mendiant, voleur et menteur, mais aussi le membre d'une confrérie dédaignée par l'islam savant. Les Gnawa sont donc marginalisés et méprisés au quotidien comme dans leurs activités religieuses. Or, depuis une quinzaine d'années, leurs troupes musicales sont invitées régulièrement à se produire sur les scènes internationales. Sous le label « musiques du monde », elles connaissent un succès considérable auprès du public occidental et, par ricochet, ce succès transforme leur statut symbolique localement; elles obtiennent une reconnaissance spectaculaire au regard du mépris dont leur communauté faisait l'objet. Le mot Gnawa est bien moins un stigmate aujourd'hui que synonyme de consécration. Toutefois, le passage du rituel à la scène, mais aussi du réseau communautaire au marché transnational des «musiques du monde» bouleverse inévitablement les rythmes musicaux et sociaux de cette communauté. Quelles sont donc ces transformations qui se sont opérées dans les pratiques poético-musicales des Gnawa 
après l'introduction de la scène dans leur trajectoire ? La scène, la modernité, mettentelles en danger le rituel, la tradition?

Les sciences sociales semblent avoir résolu cette question en se gardant d'idéaliser le passé ou le progrès et en affirmant que la culture est une "machine à fabriquer de la différence " (Warnier, 2004, 105). Or, les chercheurs qui étudient le rite de possession des Gnawa (Pâques, 1991; Lapassade, 1997; Chlyeh, 1998; Hell, 2002) l'envisagent comme un rite à but principalement thérapeutique et considèrent les concerts publics contemporains comme des dimensions superficielles, secondaires, de l'activité rituelle. Comme le note Lenclud, "certains ethnologues désignent comme traditionnels des phénomènes dont ils savent pertinemment qu'ils ne sont pas conformes à un original, qu'ils savent également ne pas exister " (1987). C'est précisément le cas ici. Dans les travaux précités, les chercheurs font directement face à une culture en changement. Ils s'attachent alors à décrire le rituel comme un univers clos et ils l'isolent du présent dans lequel il évolue. Ainsi, l'absence de cette « co-temporalité » (Fabian, 2006, 9) dans les recherches sur les Gnawa du Maroc inscrit implicitement les cérémonies Gnawa dans la «tradition» dans le sens d'une "permanence du passé dans le présent [...] d'une survivance à l'œuvre » (Lenclud, 1987).

3 Mon enquête de terrain, longue d'une dizaine d'années, sur cette communauté confrérique consiste précisément à "co-temporaliser ", à étudier, sous l'angle des trajectoires, la circulation des musiciens dans des espaces d'exécution distincts, ici et maintenant. Je tente d'y saisir la manière dont ces derniers articulent diverses activités sociales et musicales a priori très différentes: les cérémonies rituelles dans les résidences privées, les concerts publics dans les festivals au Maroc et en Europe, mais aussi les cérémonies sociales (fiançailles, mariages...) ainsi que les activités de mendicité rituelle dans la ville (Majdouli, 2007). Pour pouvoir comparer ces différentes situations et cadres de l'événement, mon approche consiste d'une part à les considérer comme des pratiques sociales tout en partant du principe que «toute activité sociale est susceptible de se prêter à plusieurs versions » (Goffman, 1991, 16), que ces versions sont interdépendantes et servent de "modèle » les unes pour les autres. D'autre part et en conséquence, j'analyse au même titre le rituel et la scène. Je les observe et décris comme des pratiques sociales ordinaires et comme des situations de communication où des individus échangent en respectant certaines règles interactionnelles (Winkin, 1996, 205). Parmi ces règles se trouve le rythme, que celui-ci soit social, poétique ou musical. Le rythme est donc envisagé ici comme une organisation collective du « temps culturel » (Hall, 1984) et comme un « engagement » en interaction (Goffman, 1974,114). Dans ce sens, si le rythme est interactionnel, il n'est pas un ensemble de règles statiques. Il est au contraire continuellement réaffirmé de «façon créatrice» (Sapir, 1968, 91) par des actes individuels de communication réintégrant graduellement les changements de rythme.

4 C'est donc à partir de la description de la lila gnawa que je tente de montrer l'importance de l'accomplissement et du respect des rythmes interactionnels comme garants de la réussite du rite de possession. Ce sont les changements de rythme qui invitent à entrer dans les différentes situations et phases rituelles. Mais à l'intérieur de ce rituel, le rythme comme interaction est également susceptible de subir des microchangements. Ces derniers sont non seulement liés au caractère intrinsèquement dynamique et "créatif » de l'interaction, mais aussi aux événements externes, en particulier l'introduction des festivals et des concerts publics à l'intérieur même du 
processus initiatique des musiciens gnawa. La description ethnographique du concert de Randy Weston et les Gnawa de Tanger au musée du quai Branly me permettra de montrer les changements de rythme (avec les passages du jazz aux musiques gnawa et vice-versa) qu'introduisent, sur la parole chantée et sur le geste rituel, l'exercice transnational d'une pratique sociomusicale.

\section{Lila, nuit rituelle des Gnawa ${ }^{3}$}

5 Chaque année, au mois de Chaabane qui précède le ramadan, certains membres de la confrérie religieuse des Gnawa, et en particulier les voyantes-thérapeutes (moqadma), organisent une cérémonie rituelle, la lila ${ }^{4}$. Celle-ci se développe dans l'espace d'une journée entière du lever du soleil au petit matin. Elle comporte généralement quatre phases ${ }^{5}$ qui se succèdent: le sacrifice (dbiha), la séance récréative (kouyou), la parade d'ouverture (l'aada) et enfin la séance de danses de possession (mlouk).

Dès le lever du jour, les préparatifs commencent. Les femmes sont responsables du nettoyage des habits et des accessoires rituels, de la préparation des repas et de la réorganisation de l'espace domestique, aménagé pour accueillir le moment rituel. Après la prière de l'asr en milieu d'après-midi, dans une cour intérieure ou autour d'un point d'eau, débute la phase du sacrifice. Dans cet espace sacralisé et préparé avec soin est disposé l'animal sacrificiel encore vivant ${ }^{6}$. Le maitre de cérémonie (maalem) et sa troupe de joueurs de crotales (qraqeb) ${ }^{7}$ sont vêtus d'habits rituels de couleur rouge et prennent place à l'endroit où aura lieu le sacrifice. Les participants apportent un brasero où brûle du benjoin qui bientôt enfumera toute la cour, accentuant ainsi l'intensité propre à cette phase du rituel. Les autres accessoires sont disposés sur le sol (baluchons, plateau divinatoire, poignards, encens...) et un bol est prêt pour recueillir le sang de l'animal. La voyante-thérapeute, commanditaire de la lila, est habillée en blanc. Elle s'installe face à l'animal sacrificiel ligoté. Elle est, lors de ce moment solennel, l'objet de tous les regards. L'assistance est placée derrière elle, face aux musiciens. D'emblée, lors du ftih rahba, la cérémonie est ouverte par les chants de louange au prophète (Sala ya nabina), le répertoire des esprits possesseurs (mlouk) ${ }^{8}$ est invoqué et en particulier les chants des esprits rouges du panthéon ${ }^{9}$ des Gnawa comme celui de « Hammouda » :

Hammouda, toi le sacrificateur Hammouda,

Hammouda, qui a ravi mon âme,

Hammouda, rends-moi grâce,

Hammouda, vois ce qui m'advient,

Hammouda, le gnawi ${ }^{10}$

Hammouda, qui blesse et guérit... [Répété 3 fois]

7 L'intensité émotionnelle et l'effervescence augmentent crescendo à mesure que s'approche le moment de l'immolation. Le sacrificateur ${ }^{11}$ se saisit alors de l'animal et l'égorge selon le rite consacré en islam. À cet instant précis, un jet du sang est recueilli dans le bol prévu à cet effet pour le déposer près de la voyante. Les musiciens entonnent alors le chant solennel du pardon et de la généreuse délivrance, l'Aafou. Ainsi tous les sens sont sollicités : visuel, sonore, olfactif et kinesthésique. Il s'agit d'un moment que mes informateurs décrivent comme hors du temps: les transes de possession ont déjà eu lieu et pour un non-initié, elles sont d'autant plus spectaculaires par leur irruption sans transition. 
8 L'effervescence du sacrifice étant retombée, un autre temps, un autre espace et un autre rythme s'ouvrent. Les musiciens quittent l'aire du sacrifice pour s'installer dans une chambre qui leur est réservée et la deuxième partie de la lila (les kouyou) peut commencer. Cette phase consiste en une série de chants et de danses sans transe effectuées par les membres de la troupe. Les participants ne sont ici que des spectateurs. C'est un moment spectaculaire et parfois dit "carnavalesque " (Dermenghem, 1954, 255 ; Lapassade, 1997, 50) avec des danses et des mimes divers se référant au contexte colonial, mais aussi à celui de l'esclavage. Lors de cette seconde période, les jeunes musiciens s'exercent, en présence des maîtres confirmés, non seulement à la répétition des chants, mais aussi aux battements des mains, à la danse comme à l'usage des crotales et pour les plus avancés d'entre eux, au guembri, luth à trois cordes et instrument maître de la musique gnawa. Cette partie récréative et formatrice, sous ses airs de légèreté, permet de mesurer les talents des musiciens et notamment leur lien avec l'audience, critère déterminant pour l'avancement de leur initiation. Cette séquence est également un échauffement et une préparation à la suivante, celle des mlouk. Les chants des kouyou sont principalement composés de louanges à Allah et son prophète, comme celui intitulé "Prière pour le Prophète " (Sallah ya nabina)...

Seigneur, je prie pour le Prophète,

L'envoyé de Dieu, mon Prophète,

Dieu et l'élu,

Réunissons-nous, esclaves et hommes libres,

Seigneur, je prie pour le Prophète... [Répété plusieurs fois]

... mais certains textes évoquent les mlouk, le Soudan et les ancêtres des Gnawa, ou encore le passé, la pauvreté et l'errance des esclaves comme le montre le chant de «L'oncle M’Bara », particulièrement explicite :

Le pauvre oncle M'Bara le Derviche,

Lui est malheureux,

Oh, Seigneur, guéris cet état, Dieu,

C'est un malheureux,

Monsieur monte sur le mulet,

Oncle M'Bara va à pied,

C'est lui le malchanceux Dieu,

Monsieur déroule le tapis,

Oncle M'Bara un drap usé,

Et M’Bara dort par terre,

Et M'Bara va pieds nus,

C'est lui le malheureux... [Répété plusieurs fois]

10 Ainsi, cette deuxième phase est une introduction à ce qui va suivre. Si elle se distingue dans l'économie rituelle par son caractère détendu et récréatif, les «invisibles » n'en sont pas moins présents.

11 La troisième période, la parade d'ouverture ou l'aada commence autour de minuit. Il s'agit d'un défilé, qui a généralement lieu dans les rues de la ville, où les participants, une bougie à la main, accompagnent les musiciens en chantant les louanges au prophète et le pardon (l'aafou) (Claisse, 2003). Pour la première et dernière fois, la troupe y utilise plusieurs grands tambours ( $t b e l)$ ainsi que les crotales. Cette phase est également unique dans la suite rituelle, car c'est le seul moment où les musiciens et les participants sont à l'extérieur, qu'ils s'adressent au quartier, aux voisins, aux profanes en d'autres termes. Ce cortège bruyant précède la partie des mlouk et a pour objet, selon 
mes informateurs, d'annoncer au voisinage la tenue de la lila dans la résidence du commanditaire, mais aussi d'appeler et d'inviter les esprits possesseurs à rejoindre l'assemblée. Si cette phase est plus courte que toutes les autres identifiées ici, elle est d'une importance capitale par son caractère public, son effet d'annonce aux Hommes et d'invitation des invisibles.

Enfin, peu après minuit, une pause est marquée et l'espace des danses de possession (généralement la salle de séjour) est sacralisé par les fumigations. La quatrième période " sérieuse » des danses de possession (mlouk) commence. Le maître de cérémonie est entouré de sa troupe face à l'assistance. Un plateau comportant les accessoires rituels est au centre de la pièce. Les danses de possession peuvent maintenant avoir lieu. Les musiciens sont en effet les principaux protagonistes de cette dernière période. Jusqu'au lever du jour, ils vont invoquer, en chantant successivement les sept familles d'esprits et de saints du panthéon des Gnawa. La troupe marque une pause rapide de bénédiction à la fin de chaque série d'invocations ${ }^{12}$ où les musiciens sont récompensés par une offrande pécuniaire. Lors de la séance sérieuse des mlouk, les musiciens doivent en effet observer la suite des chants ${ }^{13}$ et les commanditaires ainsi que les obligations rituelles : tenues, couleurs, fumigations ou accessoires spécifiques à chaque entité. Contrairement à ce qui se passe lors des kouyou, pour cette deuxième phase du rituel, l'erreur ou l'improvisation sont lourdes de conséquences. Le risque est en effet de mécontenter les entités présentes, anthropomorphisées dans les corps des participants. Des paroles mal chantées, un accessoire oublié (bols d'eau pour Sidi Moussa ou bonbons pour Lala Mira) ou encore une entité alliée à l'un des participants, mais négligée par les musiciens peuvent rompre l'économie de la cérémonie: la rupture de la voix du maitre de cérémonie, une crise chez un des musiciens ou encore, dans les cas les plus extrêmes, l'attaque d'un des membres de la troupe par un participant, sont interprétés comme la manifestation d'un esprit insatisfait.

\section{La cérémonie rituelle comme interaction sujette au changement}

L'ordre des quatre grandes périodes de la cérémonie rituelle des Gnawa présenté ici (le sacrifice dbiha, la séance récréative des kouyou, la parade d'ouverture ou aada puis les mlouk) est généralement respecté, mais il ne s'agit nullement de la description d'une lila «type ». Outre les différences régionales ${ }^{14}$, chaque «nuit » est orchestrée en fonction d'un contexte et en fonction des attentes des participants et des commanditaires. Cette nuance est au cœur de mon propos ici. Le rituel n'est pas exempt de nombreux microchangements qui sont liés à sa nature intrinsèquement changeante si elle est entendue comme un phénomène de sociabilité ordinaire.

Les suites musicales sont par exemple loin d'être une reproduction stricte et complète du répertoire. Si la lila est généralement supposée accorder une place à toutes les devises du panthéon des Gnawa, les musiciens n'en sélectionnent pas moins les chants à exécuter en fonction du contexte. Ce choix est déterminé dans et par l'interaction ellemême, dans "l'engagement» entre les participants (Goffman, 1973,114). Lors de la période récréative des kouyou, les Gnawa orchestrent finement l'ordre des chants en fonction de l'assistance, de ses attentes et parfois même de ses goûts. Ici, le contact avec l'assistance est déterminant et, dans ce sens, cette séance est tout autant récréation qu'une re-création. Le talent des musiciens est jaugé à l'aune non seulement 
de la maîtrise du rythme musical et du répertoire, mais aussi de leur relation à l'audience. Des arguments esthétiques sont avancés pour justifier ces choix notamment textuels. Certaines devises connaissent peu de modifications. Plus simples, plus rythmées, facilement mémorisables, d'une écoute plus accessible, comportant peu de paroles complexes, elles sont très souvent utilisées sur scène : "Dieu mon maitre " (Rabbi Moulay) ou encore «Il n'y a de Dieu qu'Allah» (La Ilaha illa Allah) :

Il n'y a de Dieu qu'Allah

Mes maîtres sont de la lignée de Mohammed

J'invoque le nom de Dieu, ainsi qu'il faut toujours le faire

Puis je glorifie notre Prophète, notre Seigneur Mohammed

[Répété plusieurs fois]

Mes informateurs affirment en effet connaître les préférences des commanditaires et des participants et disent qu'ils s'y adaptent systématiquement par le choix des devises comme par le jeu sur leur longueur. Les chants des Gnawa se composent en effet de plusieurs airs similaires répétés un certain nombre de fois sur le même rythme. Il est donc aisé de les prolonger ou de les raccourcir en fonction des souhaits de l'audience. Les musiciens sont récompensés par de l'argent, comme je l'ai noté plus haut, précisément lorsqu'ils parviennent à joindre une justesse et une maitrise textuelles et musicales à une compréhension fine des attentes de l'audience.

Mais que se passe-t-il lors des deux dernières phases du rituel, la parade d'ouverture et les mlouk, concernant leur répertoire poétique réputé intouchable et dont l'usage est, $a$ priori, réservé aux initiés et interdit sur scène ? Cette dernière phase du rituel semble être le lieu de la permanence. Certains Gnawa s'abstiennent d'utiliser ce répertoire sur scène. Or, de nombreux autres microchangements (verbaux et cérémoniels) y ont lieu et ne sont perceptibles qu'à travers une lecture historique du rituel et de l'observation régulière des cérémonies. Lors de l'aada notamment, les conditions urbaines, mais aussi les contraintes économiques obligent les officiants à réagencer les espaces et les temps de la cérémonie en les adaptant aux conditions de la ville. Auparavant, toute grande lila se déroulait sur trois jours (Hell, 2002, 351). Elle est aujourd'hui, sauf lors des grands moussem du mois de Chaabane, réduite à une seule nuit. Et en raison du bruit et des autorisations administratives, la parade d'ouverture se réduit à un simple défilé rapide autour ou devant l'entrée de la résidence du commanditaire. Les animaux sacrificiels onéreux (généralement des boucs) sont remplacés par des coqs ou des moutons. Tout d'abord, lors de la période des mlouk, certains esprits réputés dangereux ne sont plus invoqués comme celui de Kouria Balanda qui conduisait les danseurs à revêtir une peau animale ou encore celui de Bala Bala Dima, l'esprit-serpent (Hell, 2002, 350). D'autres chants des mlouk en revanche deviennent très populaires en particulier celui de « Lala Mira », entité féminine de couleur jaune, ou celui de "Baba Hammou », esprit puissant des abattoirs, présent sur de très nombreux disques et dont les rythmes sont appréciés par le grand public :

Je me mets sous ta protection, Baba Hammou,

o Gnawi, beauté de la séance,

Je suis au service du sacrificateur,

Je viens avec le bouc,

Et j'ai brûlé l'encens comme demandé,

Je me mets sous ta protection, Baba Hammou... [Répété plusieurs fois]

17 En écourtant la lila, le rythme musical et social se transforme inévitablement pour s'adapter aux attentes des participants et aux contraintes économiques et urbaines de l'organisation d'une cérémonie de ce type. Comme pour les kouyou, les chants des mlouk 
sont également fluctuants $\mathrm{s}^{15}$ et permettent un jeu avec les longueurs ${ }^{16}$ à travers l'écoute attentive des réactions de l'assistance. Ainsi, l'ordre rituel, aussi fixe qu'il puisse paraître, est toujours répété différemment en fonction des commanditaires et de l'assistance. La relation aux participants, au public, est donc une dimension déterminante de cet exercice musical. Le présent des musiciens imprime également sur leurs pratiques verbales, cérémonielles et musicales des variations régulées liées au caractère actuel, urbain et public de leurs expériences pratiques.

Ensuite, avec l'introduction de la scène et du disque, mes informateurs affirment abandonner certains chants du répertoire rituel. À l'instigation de l'audience, les musiciens préfèrent des devises plus populaires, car jouées régulièrement sur scène ou gravées sur les disques des maîtres reconnus. C'est le cas du chant du guérisseur ( $\mathrm{Ya}$ chafi Ya Afi) popularisé par Nass El Ghiwane, groupe musical plébiscité dès les années soixante-dix. En effet, bien avant le phénomène des musiques du monde, de nombreux musiciens, hommes de lettres et de théâtre se sont intéressés à cette communauté de musiciens. Dans les années soixante, à la suite du passage de Jimi Hendrix, Léonard Cohen, Cat Stevens, les Rolling Stones ou du Living Theatre de Julian Beck à Essaouira, une communauté de hippies s'installe dans un petit village à cinq kilomètres de la ville et assiste aux lila locales. En parallèle, le maalem Abderrahman Kirouche, dit Paco, originaire de la ville devient un des musiciens vedettes de Nass El Ghiwane. Ce groupe de musiciens a révolutionné la musique populaire locale. Produit purement marocain, il renouvelait avant l'heure les musiques et le répertoire gnawa en mêlant les répertoires et des instruments (traditionnels et modernes) à l'instar de la musique africaine contemporaine. C'est cette histoire-là qui servira de socle à la circulation internationale des Gnawa (Institut du monde arabe à Paris dans les années quatrevingt) et à la création, à la fin des années quatre-vingt-dix, du Festival Gnaoua d'Essaouira musiques du monde. Ce festival international leur permet de sortir définitivement de l'anonymat et de la marginalisation dont ils faisaient l'objet.

De plus, comme je l'ai souligné en introduction, ces musiciens se sont toujours produits, à différentes échelles, dans différents espaces d'exécution : lors des occasions sociales comme les cérémonies de mariage et aussi sur les grandes places urbaines ou dans la rue où ils ont expérimenté la rencontre avec le "grand public ». La quête, la demande publique de l'aumône par l'activité musicale fait en effet partie de la trajectoire initiatique des Gnawa. Dans le cadre de ces activités extérieures, c'est ce répertoire divertissant, « spectaculaire et carnavalesque » des kouyou qui est utilisé pour satisfaire un public plus large. Les Gnawa "font du spectacle $»^{17}$, selon leurs propres mots, trouvent dans ce répertoire un moyen de subsistance et réservent a priori aux initiés les séances où sont sollicités les esprits possesseurs. Ce répertoire divertissant, avec ses rythmes fluctuants et ses textes modulables, est précisément celui qui est utilisé sur scène. Par ailleurs, s'observent également des changements dans les codes vestimentaires et le choix esthétique des accessoires. Les musiciens réutilisent ainsi régulièrement, dans le cadre des cérémonies privées, les costumes colorés et ornés de cauris dont ils se vêtent habituellement sur scène. Les participants prêtent également une attention plus grande aux tissus et aux couleurs comme à l'esthétique des accessoires rituels. Cette observation est liée à une question d'âge et de classe sociale. Les nouveaux membres ou sympathisants des Gnawa sont aujourd'hui plus jeunes et plus aisés (en particulier à Casablanca) et n'ont aucune difficulté à déclarer publiquement leur affiliation à la culture des Gnawa. En effet, la pratique rituelle, 
autrefois stigmatisante, est aujourd'hui bien moins secrète. Au contraire, les membres et participants accentuent même parfois avec ostentation l'organisation d'une cérémonie Gnawa dans leur demeure. Aussi, l'usage des appareils d'enregistrement audiovisuel est aujourd'hui bien mieux toléré qu'auparavant. Le rite, a priori à but thérapeutique, revêt ainsi une dimension musicale, esthétique et artistique accentuée par le besoin d'en garder une trace visuelle et sonore. Cette dimension est au cœur de la question des rythmes et des changements de rythme. C'est dans ce cadre, comme je l'ai noté plus haut, que les formats, l'ordre et la succession des chants changent de manière très progressive par la mise en avant de certains airs affectionnés par l'audience. La réinvention d'un récit musical dans la temporalité du concert public (une heure environ) à travers la fluctuation de chants et de rythmes qui maintiennent l'intensité de l'action musicale et l'attention du public, ces ajustements font intimement partie de la reconfiguration des rythmes rituels sur scène. Par conséquent, cette suite permanente de microchangements ainsi que le caractère résolument dirigé vers le public des activités initiatiques et rituelles des Gnawa montrent que l'exercice scénique n'est pas inédit. Je formule donc l'hypothèse que l'aisance interactionnelle des Gnawa, déjà présente lors des cérémonies rituelles, accentuée par leur expérience sur les places publiques et leurs activités de mendicité, appuyée par le succès de groupes populaires tel que Nass El Ghiwane, trouve ici, de mon point de vue, une forme de continuité avec l'accès à la scène. Voyons à présent comment cette mécanique complexe de la cérémonie se projette sur scène et comment les répertoires et les rythmes sont modulés en direction d'un public non initié et de culture occidentale?

\section{Randy Weston et les Gnawa de Tanger : rupture ou continuité ?}

Le concert de Randy Weston et les Gnawa de Tanger du 27 mars 2009 au théatre Claude LéviStrauss est un exemple parmi d'autres des nombreux concerts donnés par des troupes de Gnawa. Il est particulièrement intéressant toutefois parce qu'il est présenté au musée du quai Branly et dans le cadre d'un événement particulier, l'exposition « Un siècle de Jazz ». Au musée des « arts premiers », la musique des Gnawa est décrite comme une référence à l'Afrique subsaharienne et par extension aux « origines » du jazz. Elle a ici le statut d'un objet ethnographique. Les Gnawa, tels qu'ils sont mis en scène dans ce contexte muséal, sont une des médiations de l'événement qui recrée ainsi une atmosphère "atemporelle" d'une culture musicale, implicitement située dans le passé, dans un autre temps, puisque précisément placée dans le cadre des « origines du jazz ».

21 En effet, avant l'entrée des musiciens, une attention particulière semble avoir été accordée à la disposition des instruments sur le plateau. Deux espaces sont distingués à même la scène : l'espace des Gnawa (avec ses tapis, poufs, couvre-chefs et guembri) et l'espace du jazz contemporain, avec la mise en avant des instruments modernes, des micros et amplificateurs. Les éléments de ce décor, de cette " mise en scène » au sens de Goffman, sont la première introduction au spectacle, et proposent d'emblée une « définition de la situation » $(1973,29)$. Cette situation permet à l'expérience musicale de présenter une certaine forme de stabilité, elle met en scène, en les idéalisant, les valeurs communément associées à une performance de ce type en distinguant ces deux espaces, ces deux territoires : celui du mobile et celui de l'« atemporel ». À l'arrivée des 
musiciens, la tenue, la gestuelle et les costumes ne font que confirmer cette opposition. Randy Weston, sous un tonnerre d'applaudissements, s'adresse au public en disant: "Gnawa music, It's not a music that changes. It's a music that we learn from. It's coming from the earth. It's a spiritual music!". Le discours de Weston donne une confirmation supplémentaire à la situation de communication déjà introduite sur le plateau par les accessoires et les éléments du décor: la différence entre deux pratiques musicales, deux temps, deux espaces qui sont amenés à se rencontrer. Le dispositif scénique, les discours, mais aussi, nous allons le voir, la structure du concert, sont donc autant de médiations, au sens d'Antoine Hennion $(1993,14)$ qui organisent le chemin entre l'œuvre et l'auditeur. La musique du jazz serait une musique présentée comme changeante et celle des Gnawa, mise en scène comme une musique qui ne change pas.

Le concert propose en effet un chemin, un récit en plusieurs actes recomposant l'histoire du jazz et de ses origines. J'emprunte ici la notion d'intensité à Richard Schechner. Celui-ci considère que chaque performance développe une structure dramatique fondée sur des accumulations et des accélérations d'intensité (Schechner, 1989, 19). En effet, le premier acte du concert au musée des Arts premiers commence par une composition originale de Weston «Tribute to Duke Ellington ». Ce morceau-hommage représente la forme contemporaine du jazz, mais il s'agit aussi d'un repère temporel : l'audience écoute religieusement le jazz tel qu'il est aujourd'hui. À la fin de ce premier morceau, Weston et sa troupe cèdent entièrement la place aux Gnawa de Tanger avec la troupe du maalem Abdellah El Gourd et Abbes Larfaoui. Ces derniers sont seuls sur scène avec leurs percussionnistes et jouent trois chants : le premier issu de la parade d'ouverture, le deuxième des kouyou et le dernier des mlouk: «Allah Ya nabina » (Allah, notre prophète) et "Sidi Moussa » (esprit possesseur de l'eau et de la mer, de couleur bleue). L'introduction des grands tambours et des danses spectaculaires des kouyou marque une véritable rupture avec le morceau précédent de Weston. Les deux chants suivants signent la fin du deuxième acte, de l'espace/temps des Gnawa et de leur musique qui «ne change pas » comme l'annonçait Weston. Le troisième acte marque le retour de Weston et de son quintette sur le devant de la scène, alors que la troupe des Gnawa se retire à nouveau. Weston précise, en guise d'introduction, qu'il s'agit de "Blue Moses», une réinterprétation du chant gnawa précédent. La recherche musicale de Weston est donc mise en avant : il est présenté comme étant allé aux sources du jazz et ayant "fusionné » le passé avec le présent. Enfin, point culminant et a priori final du concert, lors du quatrième acte, les Gnawa reviennent sur scène et les deux groupes de musiciens jouent ensemble: passé et présent sont réunis à même le plateau. Weston explique à nouveau qu'il s'agit d'une session d'improvisation ayant pour base un des chants des mlouk gnawa : "Lalla Mira », esprit féminin, faisant partie du panthéon des esprits jaunes. À la fin du morceau, certains membres du public, particulièrement séduits, montent sur scène et dansent avec le maalem El Gourd. Ensuite, tous les musiciens se retirent et la salle applaudit allégrement.

La référence à l'univers cérémoniel est présente dans ce concert à travers la scénarisation, la scénographie, les accessoires et les discours. Toutefois, peu de parenté demeure avec la lila et ses phases décrites plus haut. Dans de nombreux concerts, la parade d'ouverture introduit les spectacles musicaux. Les grands tambours et les percussions aux rythmes soutenus installent tout de suite une ambiance joyeuse et festive. Ensuite, commencent les chants des kouyou et leurs danses spectaculaires. L'ordre rituel est ainsi inversé pour les besoins de la scène. Ce concert propose un autre 
récit, une autre "version» au sens de Goffman de "présentation de soi », liée aux expériences passées des Gnawa. Aussi, l'interdit quant à l'usage du répertoire des mlouk tombe $^{18}$ comme je le décris ici, avec l'usage des chants de Lalla Mira ou de Sidi Moussa.w

Aussi, certains musiciens reconnus, devenus des vedettes nationales (Maalem Alikane, Boussou, Baqbou et bien d'autres), développent-ils à présent une véritable recherche musicale personnalisée et sont invités régulièrement à participer aux lila comme aux concerts précisément pour leur style musical singulier. Les musiciens ne sont plus ici de simples passeurs d'une "tradition» ancestrale, mais développent une personnalité musicale et une créativité propre dont le rayonnement dépasse les frontières de la confrérie et qui prouve qu'il ne s'agit plus ici d'une simple "folklorisation ». De même, si le rituel change en raison des interactions avec la scène et des contraintes économiques et urbaines, il n'en est pas moins vivace aujourd'hui au sein de la confrérie. Les musiciens développent ainsi deux trajectoires sans que celles-ci soient contradictoires. Elles deviennent au contraire interdépendantes, dans les deux sens, l'une de l'autre. Je cite l'exemple du maalem Hassan Boussou qui réside à Lille et dont le parcours est à mon sens exemplaire. Il est en effet invité régulièrement aux festivals (à Essaouira où il est membre de la direction artistique du festival, mais aussi en Europe) sans cesser pour autant d'officier dans des lila à Casablanca, Marrakech et parfois même en France (Guichenet, 2005). Au mois de Chaabane de l'année dernière, je le rencontrai au festival Diwane ${ }^{20}$ d'Alger où il se produisait avec sa troupe et m'entretins avec lui lors de notre vol retour. Le maalem Boussou, fatigué, se demandait alors comment il allait assurer le soir même une lila à Casablanca ${ }^{21}$. Ce faisant, il m'affirmait que son activité cérémonielle était tout aussi régulière que les concerts publics et qu'il était primordial pour lui de maintenir ces deux activités en parallèle. Il en allait d'une part de son alliance avec les esprits gnawa et d'autre part de cette double reconnaissance dont il bénéficiait: dans le réseau confrérique comme auprès du grand public. Les activités rituelles et festivalières se nourrissent donc mutuellement.

À l'instar de Weston, et à travers la scénarisation du concert, la posture et les costumes, les Gnawa disent et montrent également un jeu avec le temps. Leurs performances qui se jouent au présent, mais qui ont recours au passé ne sont pas nécessairement paradoxales. Comme je l'ai montré, les musiciens, dont la pratique musicale est constituée par le rapport à l'audience, y développent une compréhension fine des enjeux de la scène et des stratégies élaborées pour s'adapter à des audiences anonymes, à des contextes et des formats qui leur étaient jusque-là étrangers, mais pas totalement inconnus ${ }^{22}$. Les Gnawa sont certes attirés par la reconnaissance internationale et par les avantages économiques (bien plus importants que lors d'une lila notamment). Il n'a fallu que quelques années à ces musiciens pour s'ajuster parfaitement aux contraintes de la scène. Mon hypothèse est qu'ils y étaient précisément préparés parce que l'adaptation aux participants fait partie du processus rituel (lui-même non figé). Ainsi, leurs expériences passées, les rencontres et les associations musicales nombreuses dont leur communauté a fait l'objet donnent lieu à une forme de continuité entre rituel et scène.

En partant de l'idée que le rythme est interactionnel et en envisageant l'activité rituelle autrement que comme le lieu du " vrai » et du "légitime », je peux à l'aune des données présentées ici, considérer que le passage de la cérémonie rituelle à la scène est une coordination entre plusieurs "versions" des expériences musicales des Gnawa 
(Goffman, 1991, 30). Aucune de ces versions n'est ontologiquement plus légitime que l'autre, elles servent plutôt de modèle les unes par rapport aux autres et sont aujourd'hui interdépendantes comme le montre l'exemple du Maalem Hassan Boussou. La première version est celle de l'univers rituel où les Gnawa évoluent : la cérémonie rituelle envisagée comme une sociabilité ordinaire et le rythme musical et social comme interaction sont par essence sujets aux changements. La seconde est celle de leurs spectacles en public sur les grandes places, dans l'espace urbain ou dans les occasions sociales. C'est également dans ce cadre qu'ont lieu les rencontres avec les musiciens européens, mais aussi qu'a lieu l'évolution locale de la musique gnawa avec des groupes populaires comme celui de Nass El Ghiwane. La troisième est celle de la scène des festivals nationaux ou internationaux où les performances sont scénarisées, fusionnées mais aussi l'espace où la musique est personnalisée, renouvelée par les Gnawa eux-mêmes. Toutes ces modalités sont en dialogue, coordonnées les unes avec les autres et sont à présent constitutives de l'activité professionnelle des musiciens gnawa. L'introduction de la scène n'est donc pas conçue ici comme une opposition au rituel, les deux univers ne sont pas séparés ni hiérarchisés. Le rythme, comme organisation du temps collectif et comme recherche de la régularité interactionnelle nous force à analyser la reconstruction de la mémoire collective comme ne relevant pas de l'ordre de l'analogie, de la fixité ou de la permanence. Ce qui apparaît comme une proposition paradoxale - proposer au public au présent une musique qui a recours au passé - implique d'accepter l'idée que ce passé lui-même est en transformation puisqu'il se joue précisément au présent.

\section{BIBLIOGRAPHIE}

AYDoun, Ahmed, 1992, Musiques du Maroc, Casablanca, Éditions Eddif.

CHLYEH, Abdelhafid, 1998, les Gnaoua du Maroc : itinéraires initiatiques, transe et possession, Grenoble, Éditions La Pensée sauvage.

CLAISSE, Pierre-Alain, (2003), les Gnawa marocains de tradition loyaliste, Paris, L'Harmattan.

DERMENGHEM, Émile, 1954, le Culte des saints dans l'islam maghrébin, Paris, Gallimard.

FABIAN, Johannes, 2006, le temps et les autres : comment l'anthropologie construit son objet, Toulouse, Éditions Anacharsis.

GOFFMAN, Erving, 1973, la Mise en scène de la vie quotidienne : la présentation de soi, Paris, Éditions de Minuit.

GOFFMAN, Erving, 1974, les Rites d'interaction, Paris, Éditions de Minuit.

GOFFMAN, Erving, 1991, les Cadres de l'expérience, Paris, Éditions de Minuit.

GUILlebaud, Christine, 2010, Du rituel à la scène de festival, suivre le texte en train de se faire Inde, France -, in Claude Calame, Florence Dupont, Bernard Lortat-Jacob et Maria Manca (éds), la

Voix actée : pour une nouvelle ethnopoétique, Paris, Kimé, p. 91-108. 
HALL, Twitchell Edward, 1984, la Danse de la vie : temps culturel, temps vécu, Paris, Éditions du Seuil. HELL, Bertrand, 2002, le Tourbillon des génies : au Maroc avec les Gnawa, Paris, Éditions Flammarion. HENNION, Antoine, 1993, la Passion musicale : une sociologie de la médiation, Paris, Éditions Métailié. LAPASSADE, Georges, 1997, les Rites de possession, Paris, Éditions Anthropos.

LENCLUD, Gérard, 1987, la Tradition n'est plus ce qu'elle était..., Terrain, nº 9, p. 110-123.

MAJDOULI, Zineb, 2007, Trajectoires des musiciens gnawa : approche ethnographique des cérémonies domestiques et des festivals de musiques du monde, Paris, L'Harmattan.

PÂQUES, Viviana, 1991, la Religion des esclaves, recherche sur la confrérie marocaine des Gnawa, Bergamo, Moretti e Vitali Editori.

RENAULT, François et DAGET, Serge, 1985, les Traites négrières en Afrique, Paris, Éditions Karthala.

REYSOO, Fenneke, 1991, Pèlerinages au Maroc : fête, politique et échange dans l'islam populaire, Paris, Éditions Maison des sciences de l'homme.

Sapir, Edward, 1968, Linguistique, Paris, Éditions de Minuit.

SCHECHNER, Richard, 1989, Between Theatre and Anthropology, Philadelphia, University of Pennsylvania Press.

WARNIER, Jean-Pierre, 2004, la Mondialisation de la culture, Paris, La Découverte.

WINKIN, Yves, 1996, Anthropologie de la communication : de la théorie au terrain, Bruxelles, Éditions De Boeck Université.

\section{Discographie}

BENHAMOU-EL MADANI, Rahma, 2011, Tagnawittude, Paris, Pleins cadres [DVD, 90 mn]. Boussou Hassan Ensemble (1999), Sounds from an ancient land, Paris, Amiata Records [CD Audio]. Boussou, Hmida, 1999, Chants et musique Gnawa du Maroc, Limoges, Al Sur Productions [CD Audio].

Collectif, 1984, Maîtres Gnawa d'Essaouira, Essaouira, La Pensée sauvage [CD Audio].

Collectif, 1993, Maroc, Hadra des Gnaoua d'Essaouira (Musique rituelle), Paris, Ocora Radio France [CD Audio].

Collectif, 2003, Essaouira Festival Gnaoua, Paris, Creon Music-A3 Communication [CD Audio]. EL GNAWI, Hamid, 1999, Saha Koyo, Paris, Erato Disques [CD Audio]. GUICHENEY, Pierre, 2005, Des Gnawa dans le bocage, Le Mans, 24 images Productions [Film DVD]. NASSEL GHiwANe, 2000, Chants Gnawa du Maroc, Paris, Buda Musiques [CD Audio]. WESTON, Randy, 1995, Splendid Master Gnawa Musicians of Morocco, New York, Verve [CD Audio]. WESTON, Randy, 2000, Spirit! The Power of Music, New York, Verve/Gitanes [CD Audio].

\section{NOTES}

1. Le rite de possession des Gnawa est un adorcisme par opposition à exorcisme. Il est fondé sur l'alliance avec une entité externe (Chlyeh, 1998). 
2. Si les liens avec la traite négrière en terre d'islam et le territoire de l'ancien Soudan sont établis, les historiens, faute d'archives, peinent à démontrer les origines exactes du mot "Gnawa » lui-même, de la communauté et encore moins de sa formation comme confrérie religieuse au Maroc (Renault et Daget, 1985).

3. Pour une question de lisibilité, je décris ici l'ordre rituel d'une lila qui a eu lieu en 2009 à Casablanca.

4. La lila est également appelée moussem lorsqu'elle est organisée par une voyantethérapeute au mois de Chaabane qui précède le ramadan. Afin d'invoquer la protection divine, toutes les confréries religieuses marocaines organisent des fêtes ou moussem à certains moments du cycle calendaire, des cycles de la vie ou du cycle religieux (Reysoo, 1991, 18).

5. L'identification des périodes (et de leurs rythmes) dépend du regard porté sur elles. Le regard ethnographique reconstruit donc phénoménologiquement les temps et leurs usages. La catégorisation de la lila en phases peut donc donner lieu à de nombreuses subdivisions en fonction de la problématique de recherche. Ici la musique et le changement de l'espace cérémoniel sont le fil conducteur.

6. L'animal sacrificiel est généralement un bouc de couleur noire.

7. Qraqeb, ou crotales, instruments de percussion formés de deux plaques métalliques en forme de huit. Les percussionnistes les actionnent dans chaque main entre le pouce et le médius. Attachées par paire au moyen de liens en cuir, en les entrechoquant, sont produits tous les détails du rythme. (Aydoun, 1992, 133-138).

8. Mlouk: melk au singulier désigne, chez les Gnawa, les entités surnaturelles ou esprits possesseurs qui « prennent » les participants lors des transes.

9. Le panthéon des Gnawa se compose de sept familles de saints et d'esprits possesseurs aux sept couleurs différentes dont les participants se parent lors de la lila.

10. Gnawi, singulier de Gnawa.

11. Le Zoukay est le membre le moins initié de la troupe des musiciens, en charge du sacrifice.

12. Pour une description plus détaillée du cortège et de la partie des mlouk, cf. MAjDouli (2007, 38-53).

13. Les sept familles de mlouk sont invoqués tout au long de la phase des mlouk, une par une selon un certain ordre dicté par les usages des participants. Cet ordre diffère d'une région à l'autre.

14. Les étapes du rituel ont un ordre différent selon les régions et les familles gnawa. Par exemple, LAPASSADE et CHLYEH notent que, dans la région de Marrakech et d'Essaouira, l'aada a lieu vers minuit mais est orchestrée en première partie dans le Gherb (Fès) ou à Casablanca. (Lapassade, 1997, 49 ; Chlyeh, 1999, 25).

15. "Le soubassement rythmique des Gnawa est régulier alors que le chant est fluctuant même s'il est mesuré. Autant la ligne mélodique chantée est coulante autant la percussion est détachée [...] Le chanteur interprète donc selon son état d'âme et sa façon de sentir le rythme. » (Aydoun, 1992, 136), et j'ajouterai en fonction de sa relation à l'audience et au contexte interactionnel.

16. "La phrase musicale gnawa contrairement aux autres genres de musique populaire marocaine peut être étendue.» (Aydoun, 1992, 137). 
17. Dans son article sur les Indiens du Kerala, Guillebaud $(2010,103)$ décrit le rite de possession des dieux serpents tel qu'il a été présenté au musée du quai Branly. À l'instar des Gnawa, les indiens, dit-elle, font «comme si ». Dans mon propos et par rapport aux Gnawa, le «comme si » ou «faire du spectacle », n'est pas un jugement de valeur et n'est pas de l'ordre du vrai ou du faux. Comme le note Goffman (1991), toute " expérience ", toute "activité ", toute "situation » sociale peut se prêter à plusieurs « versions », plusieurs « cadrages ». Ceux-ci entretiennent des rapports les uns avec les autres et renvoient les uns aux autres, servent de « modèle » les uns pour les autres.

18. L'appel du public, disent mes informateurs, est plus fort que celui de l'interdit rituel.

20. Les Diwane sont les cousins algériens des Gnawa du Maroc.

21. Précisons que cette lila est dépendante de la double reconnaissance donc bénéficient les Gnawa qui - reconnus sans les festivals - sont également légitimés dans la communauté confrérique et dans leur compétence rituelle.

22. Les rituels aussi demandent bien entendu une adaptation au contexte et au publicparticipant; c'est pourquoi je considère ces deux activités comme des versions non strictement séparées.

\section{RÉSUMÉS}

Les Gnawa du Maroc connaissent depuis plus d'une dizaine d'années un bouleversement de leurs pratiques poético-musicales. À la suite de leur inscription sous le label « musiques du monde » les musiciens, autrefois « initiés ", doivent maintenant s'approprier de nouvelles compétences pour dialoguer avec la musique occidentale, mais aussi modifier le répertoire musical ainsi que la mise en scène des performances pour s'adapter à un public transnational. Le temps rituel est ainsi transformé en passant à la scène. À partir d'une approche interactionniste, je propose d'examiner ces changements sous l'angle du rythme social et musical, pour tenter de mieux comprendre les transformations pratiques et symboliques qui s'opèrent au sein de cette communauté.

For more than a decade, The Moroccan Gnawa musicians have known a disruption of their poetic and musical practices. Registered under the "world music" label, initiated musicians must now adopt new skills in order to interact with Western music. They also have to change some the musical repertoire and the staging of their performances to adapt to a transnational audience. The ritual time is thus transformed through the stage. From an interactionist approach, I propose to examine these changes in terms of social and musical rhythm, trying to understand the practical and symbolic transformations that are taking place in this community. 
INDEX

Mots-clés : rituel, scène, interactionnisme symbolique, musiques du monde

Thèmes : anthropologie de la communication

nomsmotscles Gnawa

Index géographique : Maroc

Keywords : Ritual, Stage, Anthropology of Communication, Symbolic Interactions, World Music, Gnawa of Morocco

\section{AUTEUR}

ZINEB MAJDOULI

GRS, UMR 5040, Université catholique de Lille 